\title{
VIOLÊNCIA SEXUAL NO NAMORO: RELEVÂNCIA DA PREVENÇÃO ${ }^{1}$
}

\author{
Sónia Caridade \\ Carla Machado ${ }^{2}$
}

Resumo: Este artigo apresenta uma revisão da investigação sobre a violência sexual ocorrida no contexto das relações de namoro. Para esta revisão, foi efectuada uma pesquisa sistemática nas revistas publicadas entre 1985 e 2006 nas bases de dados PsycARTICLES, PsycINFO1887, Sociology: A SAGE Full-Text Collection, EBSCO-HOST: Research Databases e IBSS - International Bibliography of the Social Science, para além da análise de livros publicados no mesmo período sobre o tema. A partir desta revisão, são apresentados indicadores de prevalência da violência sexual e os principais factores de risco para a sua ocorrência e caracteriza-se a cultura de prevenção existente a este nível. Especificamente, procura-se identificar as diferentes acções preventivas desenroladas neste âmbito, assim como a sua eficácia. Para concluir, reflecte-se sobre algumas limitações dos programas preventivos existentes na área e algumas recomendações são avançadas para futuras acções preventivas a desenvolver junto dos jovens.

Palavras-chave: violência sexual, namoro, prevenção, jovens.

Dating sexual violence: Relevance of prevention (Abstract): This article presents a systematic review of the current body of literature about sexual violence in dating relationships. This review was based on the articles published during the 1985-2006 period in the databases PsycARTICLES, PsycINFO1887, Sociology: A SAGE Full-Text Collection, EBSCO-HOST: Research Databases e IBSS - International Bibliography of the Social Science, as well as on the analysis of books published during the same period. Based on this revision, this text presents the prevalence rates of sexual violence between dating partners and analyses its risk factors. The prevention culture on this topic is also described, exploring the different kinds of preventive actions and their efficacy. In conclusion, reflections about the limita-

\footnotetext{
${ }^{1}$ Este estudo foi desenvolvido no âmbito do projecto "Violence in dating relationships" (PTDC/PSI/65852/2006), financiado pela Fundação para a Ciência e a Tecnologia.

Toda a correspondência sobre este artigo deverá ser remetida para: Carla Machado. Departamento de Psicologia, Instituto de Educação e Psicologia. Campus de Gualtar. 4710 Braga. Telef.: 253604 267; Fax: 253678 987; e-mail: cmachado@iep.uminho.pt.

2 Departamento de Psicologia, Instituto de Educação e Psicologia, Universidade do Minho.
} 
tions of the prevention program conduced in this area are made and some suggestions for future actions with youth populations are presented.

Keywords: sexual violence, dating, prevention, youngsters.

\section{Introdução}

A violência sexual nas relações de namoro, ainda que algo marginalizada nos discursos sociais e educativos, tem recebido grande atenção por parte da comunidade científica, especialmente a partir dos anos 80 e 90 . A proliferação de estudos internacionais realizados neste âmbito é um comprovativo disso mesmo (e.g., Foshee, 1996; Jackson, Cram \& Seymour, 2000; Poitras \& Lavoie, 1995). Tal literatura tem vindo a documentar não só a elevada incidência e prevalência do fenómeno, mas também o impacto e as consequências nefastas que este tipo de experiências abusivas poderá acarretar para as suas vítimas.

As investigações neste domínio têm vindo centrar-se no contexto universitário (e.g., Breitenbecher, 2000; Schwartz \& DeKeseredy, 1997), indicando que aproximadamente $50 \%$ das estudantes já experimentaram alguma forma de coerção sexual. Aliás, alguns autores (e.g., Gidycz, Hanson \& Layman, 1995) defendem que os indicadores de vitimização sexual entre as estudantes universitárias são três vezes mais elevados do que os registados na população em geral. Este tipo de vitimação parece, contudo, não ser exclusivo deste grupo populacional e evidenciar-se igualmente em grupos etários mais novos (e.g., Jackson, Cram \& Seymour, 200; Poitras \& Lavoie, 1995). Outros estudos corroboram esta realidade (e.g., Abbey, Ross, McDuffie \& McAuslan, 1996; Gidycz, Coble, Latham \& Layman, 1993) e defendem que a agressão sexual é um problema generalizado, quer entre os adolescentes ao nível do ensino secundário, quer entre estudantes universitários.

Tais dados atestam a amplitude do fenómeno, denunciando a urgente implementação de estratégias preventivas neste domínio. Este artigo pretende contribuir para tais esforços, através da revisão da literatura disponível sobre a vitimação sexual ${ }^{3}$ perpetrada nas relações amorosas, assim como sobre os projectos de prevenção desenvolvidos nesta área.

\footnotetext{
${ }^{3}$ Apesar de não existir uma definição consensual de vitimação sexual na literatura sociológica e psicológica, todos os autores são unânimes quanto a estarmos perante uma comduta sexual não consentida (e.g., Bohmer \& Parrot, 1993; Schwartz \& Pits, 1995, cit. Schwartz \& DeKeseredy, 1997). Assim, se procurarmos uma definição ampla, poderemos considerar que a violência sexual integra qualquer tipo de contacto sexual sem consentimento, desde a violação ao controlo dos direitos reprodutores (Abraham, 1999, cit. Mahoney, Williams \& West, 2001).
} 
Esta revisão baseia-se numa pesquisa sistemática nas revistas publicadas entre 1985 e 2006 nas bases de dados PsycARTICLES, PsycINFO1887, Sociology: A SAGE Full-Text Collection, EBSCO-HOST: Research Databases e IBSS - International Bibliography of the Social Sciences, para além da análise, necessariamente menos exaustiva, dos livros publicados neste mesmo período. Para efectuar tal pesquisar utilizaram-se algumas palavras-chave, como: "sexual violence", "date rape", e "sexual coercion".

\section{Violência sexual: da prevalência à relevância preventiva}

A literatura evidencia que, frequentemente, os jovens não percepcionam as agressões perpetradas no âmbito das suas relações amorosas como abusivas e, mais especificamente, não reconhecem as relações sexuais forçadas como uma forma de violação (Muehlenhard, 1988, cit. Kuffel \& Katz, 2002). Tal tipo de representação do fenómeno resulta no típico sub-relato deste tipo de agressões, sobretudo nos casos em que o agressor é o namorado ou alguém conhecido (cf. Humphrey \& White, 2000; Vicary, Klingaman \& Harkness, 1995). Neste sentido, Hall e Barongan (1997) salientam que o comportamento sexualmente agressivo é produto de uma cultura onde este tipo de acto é tendencialmente consentido e aceite. A tolerância e legitimação desta forma específica de abuso, bem como a vergonha que muitas vezes lhe está associada, dificultam o adequado conhecimento desta realidade, bem como a sua erradicação, na medida em que reduzem a probabilidade de haver denúncia e sanção dos ofensores (Frese, Moya \& Megías, 2004).

Ainda assim, a literatura internacional comprova que a violência sexual entre adolescentes não é um fenómeno raro (e.g., Jezl, Molidor \& Wrigth, 1996; Muram, Hostetler, Jones \& Speck, 1995), incluindo a violação (e.g., Muehlenhard \& Linton, 1987). Este tipo de violência é, na grande maioria dos casos, cometido por um conhecido e, de forma frequente, pelo parceiro amoroso (e.g. Gross, Winslett, Roberts \& Gohm, 2006; Himelein, 1995; Muram et al., 1995). Um dos estudos mais frequentemente referenciado na literatura (Koss, Gidycz \& Wisniewski, 1987, cit Foshee, 1996) comprova que entre $20 \%$ a $50 \%$ das estudantes universitárias já foram alvo de algum tipo de coerção sexual no contexto das suas relações amorosas. Percentagens similares foram encontradas no estudo de Abbey e colaboradores (1996): numa amostra de 1.160 mulheres, 59\% tinham experimentado algum tipo de agressão sexual, sendo que destas $23 \%$ relataram violação e $8 \%$ tentativa de violação. Na grande maioria dos casos (95\%), as agressões sexuais foram cometidas por um conhecido da vítima (geralmente, o parceiro amoroso). Himelein (1995), através da realização de um estudo longitu- 
dinal com mulheres, comprova esta realidade, ao constatar que $52 \%$ das participantes já tinham sido alvo de algum tipo de violência sexual no contexto das suas relações amorosas, ao longo da vida. Este estudo permitiu ainda caracterizar a vitimação consoante a sua gravidade, tendo-se registado $19 \%$ de casos de vitimação menor (contacto sexual), $22 \%$ de vitimação moderada (coerção sexual ou tentativa de violação), atingindo a vitimação mais severa (violação) os $11 \%$.

Dados mais alarmantes resultam de uma outra investigação que, ao estudar a incidência das diferentes formas de agressão sexual praticadas em contexto de namoro, apurou que $77,6 \%$ das participantes femininas e $57,3 \%$ dos participantes masculinos estiveram envolvidos em algum tipo de agressão sexual e que $15 \%$ de mulheres e $7 \%$ de homens tinham sido vítimas de violação nas suas relações íntimas (Muhlenhard \& Linton, 1987).

Numa investigação levada a cabo com 600 adolescentes com idades compreendidas entre os 15-19 anos, foi possível verificar que $64 \%$ dos participantes da amostra tinham praticado ou experimentado algum tipo de pressão ou coerção sexual nas suas relações amorosas. Dos participantes vitimados por este tipo de práticas sexuais, $65 \%$ pertenciam ao sexo feminino e $42 \%$ ao sexo masculino (Serquino-Ramiro, 2005). Por sua vez, num estudo de Vicary e colaboradores (1995), 35\% das participantes femininas referiram ter sido vítimas de algum tipo de agressão sexual por volta dos 17 anos.

Os dados acerca dos crimes provenientes dos Census Bureau (2001, cit. Maxwell, Robinson \& Post, 2003) evidenciam ainda que os adolescentes com idades compreendidas entre os 12-17 anos de idade representam $30 \%$ das vítimas de violência sexual conhecidas e $20 \%$ dos ofensores. Também as estatísticas da justiça portuguesas identificam claramente os escalões etários mais jovens (até aos 29 anos) como os mais representados entre as vítimas de agressão sexual, numa proporção que era, em 1998, de 64\% do volume total de vítimas de crimes sexuais (GEPMJ, 1998). De uma forma mais específica, as estatísticas criminais informam que os menores de 14 anos são alvo de $38,6 \%$ do total dos crimes sexuais, que os jovens entre os 15 e os 19 anos correspondem a $9,7 \%$ das vítimas e que os jovens adultos entre os 20 e os 29 anos representam 13,4\% do total de vitimações (GEPMJ, 1997).

Num estudo representativo com estudantes universitários, Koss e Cleveland (1997) constataram que 53\% dos estudantes do sexo feminino já tinham experimentado algum tipo de coerção sexual, $12 \%$ revelaram ter sido alvo de tentativa de violação e $15 \%$ de violação consumada. Uma síntese de 34 estudos realizados com jovens estudantes revela que a prevalência da vitimação sexual entre as raparigas atinge os 12\% (Spitzberg, 1999, cit. Maxwell et al., 2003). Os estudos prospectivos (e.g., Gidycz, Coble, 
Lathan \& Layman, 1993; Gidycz, Hanson \& Layman, 1995) corroboram estes dados e estimam que a vitimação sexual das estudantes universitárias se situará entre os $18 \%$ e os $27 \%$.

Pesem embora os inúmeros estudos que documentam a prevalência do fenómeno no contexto universitário, será de destacar que este tipo de vitimação parece iniciar-se mais precocemente, na adolescência ou aquando do ensino secundário. Na verdade, os estudos empíricos (e.g., Bergman, 1992; Feltey, Ainslie \& Geib, 1991; Foshee, 1996) revelam que, e dependendo do tipo de actividade sexual não desejada considerado, a vitimização sexual entre os adolescentes envolvidos em relações amorosas poderá situar-se entre os $12 \%$ e os $76 \%$. Uma outra investigação envolvendo estudantes ao nível do secundário constatou que $77 \%$ das participantes femininas envolvidas em relações amorosas já tinham experimentado actividades sexuais não desejadas (Jackson, Cram \& Seymour, 2000). Tendo por base 3 amostras recolhidas em períodos distintos (1993, 1995 e 1997), Schubot (2001) verificou que a prevalência da violação no namoro entre as adolescentes, ao nível do ensino secundário, poderá situar-se entre $11,8 \%$ e os $14,9 \%$.

Grande parte destes episódios de vitimação parece ocorrer em contextos relacionais de intimidade, nomeadamente em relações de namoro ou dating 4 (Koss, Dinero, Seibel \& Cox, 1988). De facto, Koss, Gidycz e Wisniewski (1987, cit. Koss \& Cleveland, 1997) referem que 8 em cada 10 vítimas de violência sexual conhecem os perpetradores, sendo que $57 \%$ dos abusos ocorrem durante uma relação amorosa. Um outro estudo de Day (1994) evidencia ainda níveis mais preocupantes de violência e revela que $45 \%$ das violações que envolvem estudantes universitárias ocorrem no seio de relações amorosas. Outros autores (cf. Feltey, Ainslie \& Geib, 1991; Jewkes, 2002) comprovam que, efectivamente, é na intimidade do namoro que este tipo de vitimação tem maior relevo, reforçando a necessidade de implementar esforços preventivos junto desta população.

Como é sabido, a violência nas relações íntimas não se circunscreve à dimensão sexual, podendo envolver múltiplas e variadas formas, entre as quais abuso físico e psicológico. Se relativamente a estas últimas formas de violência existem algumas evidências (e.g., Machado, Matos \& Moreira, 2003; Paiva \& Figueiredo, 2004) que sugerem que esta violência pode ser mais simétrica do que tradicionalmente se pensa (ambos os géneros poderão não só constituir-se como vítimas, mas também como perpetradores), o mesmo não é tão claro no que concerne à agressão sexual. A este nível, parece existir na literatura algum consenso quanto à maior vitimação femi-

\footnotetext{
${ }^{4}$ Dada a inexistência de tradução para o termo e a sua não total correspondência ao conceito de namoro, optamos por deixar a designação no original.
} 
nina e maior agressão masculina (e.g., Bergman, 1992; DeKeseredy \& Schwartz, 1997; Foshee, 1996; Jackson \& Davis, 2000; Mahoney, Williams $\&$ West, 2001). Os estudos desenvolvidos junto de elementos do sexo masculino no ensino universitário (e.g., Abbey et al., 2001; Muehlenhard \& Linton, 1987) apontam para indicadores de agressão sexual entre os $25 \%$ e os $75 \%$, sendo que entre $7 \%$ e $15 \%$ revelaram ter cometido actos compatíveis com a definição legal de violação. Num outro estudo ainda mais recente (Loh, Gidycz, Lobo \& Luthra, 2005) realizado com elementos do sexo masculino, $31 \%$ dos participantes admitiram a prática de comportamentos sexualmente agressivos, sendo que, destes, $27,5 \%$ reconheceu ter exercido violência sexual moderada e $3,7 \%$ violência sexual severa. Outros autores sugerem que a vitimação sexual ou a violação no namoro poderá situar-se entre os $3 \%$ e os $23 \%$, no caso das raparigas, e entre $2 \%$ a $4 \%$, no caso dos rapazes (e.g., Bergman, 1992, Davis, Peck \& Storment, 1993, cit. Lanier, 2001).

Estas diferenças de género são igualmente confirmadas no estudo de Foshee (1996), no qual a vitimação feminina foi regista em $15 \%$ dos casos e a masculina em 7\%. É, contudo, de referir que outros resultados menos discrepantes em termos de género existem na literatura, revelando percentagens de homens vitimados sexualmente mais elevadas do que as habitualmente registadas. Neste sentido, destacamos o estudo de Jezl e colaboradores (1996) que, numa amostra com estudantes do ensino secundário, apuraram que $14,7 \%$ dos participantes envolvidos em relações amorosas já tinham tido uma experiência de agressão sexual. Neste estudo, ainda que as mulheres $(17,8 \%)$ se assumam mais frequentemente como vítimas deste tipo de abuso, comparativamente com o sexo oposto $(11,4 \%)$, as diferenças de género já não se mostram tão acentuadas como outros estudos evidenciam.

Na verdade, alguns autores (e.g., McConaghy \& Zamir, 1995, cit. Russell \& Oswald, 2001; Poitras \& Lavoie, 1995) têm defendido que homens e mulheres experimentam níveis similares de coerção sexual nas suas relações amorosas, reconhecendo, todavia, que a investigação da coerção sexual praticada pelas mulheres é mais limitada. Poitras e Lavoie (1995), no seu estudo sobre coerção sexual, constataram que $54,1 \%$ das mulheres que integravam a amostra tinham sido alvo deste tipo de vitimação e apenas 13,1\% dos participantes masculinos admitiram o mesmo. Ainda assim, aqueles autores defendem que, tal como ocorre com as raparigas, os rapazes também podem sofrer vitimação sexual, sendo possível a perpetração feminina de actos sexualmente abusivos. Mais recentemente, um estudo exploratório realizado por Russell e Oswall (2001) confirma que um número substancial de mulheres (18\%) adopta comportamentos sexualmente coercivos para com o sexo oposto, sendo isto mais provável em 
mulheres com história de vitimização sexual. Ainda assim, será conveniente salientar que tais indicadores, mesmo que correspondam à realidade, não transformam, por si só, o fenómeno da vitimação sexual em algo simétrico em termos de género: as formas de coerção, capacidade de imposição da força e competências de resistência da vítima não são seguramente semelhantes entre homens e mulheres, fruto das diferenças biológicas que os separam, bem como dos diferentes processos de socialização em relação ao uso da força e à sexualidade.

No que concerne à realidade portuguesa, é de referenciar que só muito recentemente se começaram a encetar esforços de investigação neste domínio, sendo ainda parcos os estudos específicos sobre a temática da violência sexual em geral ou sobre a violação perpetrada no contexto das relações amorosas. Ainda assim, é possível destacar alguns estudos recentes, tais como a investigação levada a cabo por Paiva e Figueiredo (2004), em que se procurou analisar a problemática do abuso ocorrido na intimidade dos jovens adultos portugueses. Os seus resultados sugerem que a coerção sexual, tanto na forma perpetrada $(18,9 \%)$ como sofrida $(25,6 \%)$, é o segundo tipo de abuso mais prevalente na amostra ${ }^{5}$.

A literatura clarifica, deste modo, que a violência sexual ocorre em muitas relações amorosas juvenis, fundamentando a necessidade de promover esforços preventivos dirigidos a esta população específica. Estando conscientes dos indicadores de prevalência do fenómeno, o planeamento e o desenvolvimento de acções preventivas implicam também, a nosso ver, uma compreensão detalhada dos factores de risco potenciadores da vitimação sexual.

\section{Factores de risco para a vitimação e perpetração de agressões sexuais}

Um amplo corpo de estudos tem procurado identificar os factores de risco $^{6}$ associados, quer à vitimação sexual, quer à perpetração deste tipo de agressões. De um modo geral, os factores de risco podem ser distribuídos

\footnotetext{
${ }^{5}$ Um outro estudo, realizado por Machado e colaboradores (2003) sobre a violência nas relações amorosas num campus universitário, apurou $1,3 \%$ de casos de prática de actos sexuais forçados, sendo que a taxa de perpetração destes actos rondava os $0,3 \%$. É, contudo, provável que esta percentagem relativamente baixa se devesse à natureza do instrumento utilizado no estudo, centrado sobretudo na violência física e emocional e em que apenas um item abordava directamente a vitimação sexual.

${ }^{6}$ Os factores de risco referem-se a comportamentos ou condições que possibilitam ou facilitam a ocorrência ou o contacto com um determinado problema (Sugarman \& Hotaling, 1989, cit. Harned, 2002), no caso específico, a violência sexual.
} 
por três categorias distintas: características da vítima (e.g., idade, experiências prévias de vitimização sexual, atitudes e comportamentos), características dos ofensores (e.g., idade, género, atitudes, traços de personalidade, modelos de comportamento sexual) e características situacionais (e.g., consumo de álcool, contexto de ocorrência do incidente) (Marx, Victoria \& Gross, 1996).

Começando a nossa abordagem pela análise das características da vítima, não podemos deixar de destacar que, na perspectiva de alguns autores (e.g., Schwartz \& DeKeseredy, 1997), uma ênfase nesta dimensão poderá comunicar a atribuição àquelas de alguma responsabilidade pelo abuso sofrido. Não sendo este, obviamente, o nosso propósito, consideramos que a identificação dos factores de risco que poderão colocar a mulher numa situação de maior vulnerabilidade à vitimação sexual é pertinente para o delinear de estratégias preventivas mais eficazes (Wilson, Calhoun \& Bernat, 1999).

Assim, no que concerne aos grupos de risco, vários autores (e.g., Gidycz, Coble, Latham \& Layman, 1993; Gidycz, Hanson \& Layman, 1995) consideram, com base em indicadores estatísticos, que os estudantes universitários se destacam, quer pelo risco de ocorrência de violação, quer para qualquer outra forma de vitimação sexual, comparativamente com a população em geral. A adolescência tem também sido considerada como um período de grande vulnerabilidade para a violência sexual, dada a imaturidade emocional, inexperiência relacional e iniciação à sexualidade que geralmente caracteriza esta etapa desenvolvimental (Serquino-Ramiro, 2005). O risco parece ser acrescido nos adolescentes mais novos (por volta dos 15 anos de idade), altura em que se estabelecem usualmente as primeiras relações amorosas (Vicary, Klingaman \& Harkness, 1995). Um estudo longitudinal realizado por Humphrey e White (2000) comprovou igualmente que a adolescência constitui um período de grande risco para a primeira vitimização sexual e que, por sua vez, esta poderá predizer a vitimação futura (por exemplo, no contexto universitário). Esta maior susceptibilidade desenvolvimental da adolescência é corroborada por outros autores (e.g., Black \& Weisz, 2003; Wolfe, 1994, cit. Jackson, Cram \& Seymour, 2000), que destacam também o impacto que a conformidade com os papéis de género tradicionais (e.g., homens controladores e mulheres submissas) poderá ter no envolvimento em relações abusivas.

A par da idade, a história de vitimação sexual passada tem sido consensualmente apontada pela investigação empírica como um dos factores preditores mais consistentes da vulnerabilidade feminina à vitimação sexual na idade adulta (e.g., Breteinbecher \& Gidycz, 1998, 1999; Gidycz et al., 1993, 1995, Hanson \& Gidycz, 1993; Norris, Nurius \& Dimeff, 1996; Sanders \& Moore, 1999). As experiências prévias de vitimação sexual, na 
infância (e.g., Greene \& Navarro, 1998; Humphrey \& White, 2000; Vogel \& Himelein, 1995) e adolescência (e.g., Gidycz et al., 1993; Himelein, 1995), têm sido associadas à vitimação sexual posterior. Num estudo prospectivo, Gidycz e colaboradores (1993) verificaram que uma mulher com história de tentativa de violação ou mesmo violação na adolescência tinha duas vezes mais probabilidade de experimentar uma agressão sexual. Estes resultados foram corroborados na replicação de um outro estudo conduzido por Gidycz, Hanson e Layman (1995), que comprovaram que a agressão sexual tende a ser um trauma re-experienciado. De forma análoga, também Himelein (1995) apurou que a experiência precoce de violência sexual no namoro constitui um importante preditor da vitimação sexual posterior entre as estudantes do ensino secundário. A par desta constatação, Sanders e Moore (1999) acrescentaram que existem outras experiências igualmente stressantes e negativas (e.g., maus tratos) que poderão contribuir para a vulnerabilidade feminina à violação no namoro. De acordo com estes autores, as mudanças psicológicas produzidas pelo trauma (e.g., dissociação, depressão, irritabilidade, comportamento sexual disfuncional) medeiam a relação entre as experiências negativas precoces e a vitimação posterior.

Efectivamente, torna-se fundamental compreender os mecanismos explicativos desta revitimização sexual, bem como identificar os factores que podem diminuir o risco desta suceder. As múltiplas teorias neste âmbito destacam os factores ambientais, processos de aprendizagem disfuncionais, depressão e ansiedade, estilos de coping, suporte parental e envolvimento na psicoterapia como sendo variáveis que poderão, de alguma forma, mediar a relação entre várias experiências de vitimização sexual (cf. Breitenbecher \& Gidycz, 1998). Gidycz e colaboradores (1993) constataram que o comprometimento do ajustamento psicológico (e.g., depressão, ansiedade), decorrente da vitimização precoce poderá predizer significativamente a vitimização sexual na idade adulta. Por sua vez, Norris e colaboradores (1996) verificaram que as mulheres com história de vitimação sexual experimentam algumas dificuldades psicológicas (e.g., embaraço, medo da rejeição masculina) que as impedem de demonstrar resistência efectiva face à ameaça de revitimização sexual. Assim, ainda que a mulher experimente desconforto nas interacções sexuais coercivas, a sua dificuldade em responder adequadamente à situação impede-a de sair do contexto abusivo (Wilson, Calhoun \& Bernat, 1999). Cabe, contudo, destacar que ainda que estes estudos retrospectivos sustentem a existência de uma relação entre sequelas psicológicas e revitimização sexual, as conclusões a este nível são ainda pouco consistentes e careceriam de corroboração por estudos prospectivos (Breitenbecher \& Gidycz, 1998).

Apesar de esta ser a linha de estudos mais significativa no que concerne à vulnerabilidade da vítima, uma revisão recente da investigação 
(Maxwell et al., 2003) defende abordagens que nos parecem mais questionáveis, nomeadamente a ideia de que as vítimas podem prevenir o ataque $\mathrm{e}$ que, muitas vezes, a agressão sexual é da sua responsabilidade. Esta linha de raciocínio tem já uma "longa" história, desde o estudo de Amir (1971 cit. Marx et al., 1996) sobre as "violações precipitadas pela vítima" e as numerosas críticas de que este foi alvo. Entre estas, a mais conhecida foi a acusação de que tal abordagem mais não faz do que blame the victim, fazendo eco dos mitos e estereótipos difundidos no senso comum. Neste sentido, as umlheres que não se comportam de forma consistente com os estereótipos culturais, por exemplo, que possuam um estilo de vestir entendido como "provocador" tendem a ser responsabilizadas pelos ataques sexuais (e.g., Forbes \& Adams-Curtis, 2001; Muehlenhard, Friedman \& Thomas, 1985).

Tais críticas não têm, contudo, dissuadido um conjunto de autores (e.g., Muhlenhard, Friedman \& Thomas, 1985; Muehlenhard \& Linton, 1987) de analisar a possível relação entre os comportamentos e/ou atitudes da vítima e a ocorrência de violência sexual nas suas relações amorosas. Os resultados destas investigações sinalizam que o risco de vitimização sexual feminina aumenta em função de várias situações, designadamente quando são as mulheres a iniciar a relação de namoro, quando é o parceiro que custeia os gastos da relação, quando a mulher frequenta o apartamento do companheiro e, ainda, em função da forma como a mulher se veste. Um estudo longitudinal realizado por Himelein (1995) revela também que as mulheres sexualmente mais conservadoras possuem um menor risco de serem vitimizadas sexualmente, dado que isto fomenta a desconfiança na relação amorosa e o menor envolvimento sexual. Neste sentido, o número de parceiros amorosos (e.g., Abbey et al., 1996; Maxwell et al., 2003; Shapiro \& Schwarz, 1997; Söchting et al. 2004) e a frequência de experiências sexuais consentidas (e.g., Himelein, 1995; Shapiro \& Schwarz, 1997) e relações sexuais de tipo casual (e.g., Harned, 2002) poderão igualmente representar factores de risco para a vitimação sexual.

Este tipo de dados poderá, pelo menos parcialmente, ser explicado pelo tipo de interpretações e atribuições feitas relativamente ao comportamento feminino menos convencional, no contexto de uma sociedade com um duplo padrão em matéria sexual. Neste sentido, Cook (1995) verificou, num estudo sobre atitudes face à agressão sexual, que, ainda que de uma forma global os participantes tenham afirmado não concordar com comportamentos sexualmente coercivos, em três situações (quando a mulher inicialmente concorda com o acto sexual, mas depois acaba por o rejeitar; quando "provoca" sexualmente o companheiro e quando já manteve relações sexuais anterior com aquele) a agressão sexual era desculpabilizada pelos sujeitos. Outro estudo mais recente (Geiger, Fischer \& Eshet, 2005) confirma que os jovens continuam a manifestar estereótipos desculpabiliza- 
dores da coerção sexual e, ainda que os homens surjam como os mais legitimadores deste tipo de atitudes (sobretudo ao nível da culpabilização da vítima), as mulheres não estão imunes à socialização sexista. Neste estudo, $15 \%$ das participantes femininas revelaram aceitar algumas atitudes legitimadoras da violação e de responsabilização da vítima. Outros estudos (e.g., Mahoney, Willims \& West, 2001) documentam também as atitudes sociais de responsabilização da vítima pelo problema (e.g., provocação, precipitação e ausência de precaução).

Finalmente, outros factores são referidos na literatura, ainda que de modo mais periférico, como potenciais preditores da vitimação sexual, nomeadamente o baixo estatuto socioeconómico da mulher enquanto gerador de vulnerabilidade à agressão sexual perpetrada por estranhos e problemas mentais e emocionais da vítima (cf. Söcthing et al., 2004). Um estudo longitudinal (Vicary et al., 1995) sugere também que as raparigas que consideram possuir poucas amizades e que têm uma imagem de si negativa tendem a aceitar mais facilmente condutas sexuais agressivas por parte dos seus parceiros amorosos. De igual modo, a vulnerabilidade psicológica (e.g., depressão e ansiedade) poderá predizer a ocorrência de incidentes abusivos (Green \& Navarro, 1997).

Desta forma, independentemente das polémicas assinaladas, parecem existir algumas evidências de um risco diferencial de vitimação entre as mulheres e que este poderá, pelo menos em parte, estar ligado a aspectos comportamentais da vítima, eventualmente de natureza sexual. Uma correcta e ponderada apreciação destes resultados apenas é possível, a nosso ver, se separarmos a argumentação moral e a tendência para aproveitar tais dados para culpar a vítima, centrando-nos na identificação dos contextos ou condutas que aumentam o risco e na forma de o minimizar. Uma correcta análise destes resultados parece sugerir que há comportamentos da vítima (e.g., envolvimento em relacionamentos variados) que, por meras razões estatísticas, aumentam a sua exposição a potenciais parceiros violentos, enquanto outros factores de risco (e.g., modo de vestir) dependem sobretudo da interpretação e valoração social que é feita de determinadas condutas da mulher, frequentemente imbuídas de estereótipos e distorções sobre a sexualidade e o género.

Não obstante a pertinência da identificação das características da vítima que poderão conduzir a uma maior susceptibilidade de agressão sexual, tem sido defendido que a forma mais efectiva para combater o fenómeno da violência sexual será identificar os preditores do comportamento sexualmente agressivo. Como variáveis mais susceptíveis de conduzir a comportamentos sexualmente agressivos, destacam-se: o género e as expe- 
riências de socialização, as crenças e atitudes em torno da sexualidade, a personalidade e o consumo de álcool (Loh, Gidycz, Lobo \& Luthra, 2005).

De entre os diferentes factores de risco para a agressão sexual masculina, é praticamente consensual que o sexo masculino constitui o principal grupo de risco para a perpetração de violência sexual. Uma revisão recente da literatura (Carr \& Van Deusen, 2004) sugere que esta relação poderá ser explicada pela socialização diferenciada dos papéis sexuais e pelas concomitantes crenças e atitudes diferenciadas em relação à sexualidade e à agressão. Assim, tem sido defendido que os rapazes tendem a manifestar uma percepção distinta das raparigas, no que concerne à violência sexual, decorrente da forma como absorvem os mitos ${ }^{7}$ associados à violação e as atitudes sexistas tradicionais emergentes do discurso social (cf. Marx et al., 1996). Muehlenhard e Linton (1987) comprovam estas diferenças de género, constatando que os homens sexualmente agressivos tendem a aceitar mais facilmente os papéis de género tradicionais, a violência contra a mulher e uma representação hostil ou conflitual da sexualidade. A revisão da literatura efectuada por Feltey, Ainslie e Geib (1991) ilustra também a importância do género na determinação das atitudes acerca da violação e da agressão sexual em geral, com os homens a legitimarem mais frequentemente os mitos sexuais e os comportamentos sexualmente coercivos. Por sua vez, Truman, Tokar e Fischer (1996), procurando estabelecer uma relação entre os papéis de género masculino e a violação na intimidade, constataram que os homens que aderem mais aos papéis tradicionais de género tendem, igualmente, a corroborar as atitudes e crenças legitimadoras da violência sexual. Das diversas variáveis associadas à masculinidade analisadas pelos autores, as atitudes contra as mulheres emergem como o preditor mais consistente das atitudes e crenças legitimadoras da violação nas relações amorosas. A aceitação dos mitos sobre a violação sexual surge, assim, consistentemente referenciada nos estudos empíricos como estando associada ao comportamento sexualmente agressivo, nomeadamente nos grupos de estudantes (Loh et al., 2005; Lonsway \& Fitzgerald, 1994). Efectivamente, de entre as diferentes variáveis demográficas (e.g., género, idade, etnia, educação ou ocupação) analisadas, o sexo dos participantes emerge como a variável que possui uma relação mais consistente com os mitos associados à violação (Lonsway \& Fitzgerald, 1994).

\footnotetext{
${ }^{7}$ Os mitos associados à violação podem ser entendidos como "atitudes ou crenças, geralmente, falsas, que são toleradas de forma persistente, por uma grande percentagem da população e que servem para negar ou justificar a agressão sexual masculina contra as mulheres" (Lonsway \& Fitzgerald, 1994, p. 134).
} 
Este tipo de resultados tem sido central para diversas perspectivas teóricas explicativas da agressão sexual, nomeadamente as abordagens feministas e a teoria da aprendizagem social.

Nas abordagens feministas, a agressão sexual é vista como o resultado da normal socialização masculina (e.g., Rozee, 1993), sustentando-se que certas formas de condicionamento sociocultural poderão incrementar o risco de ocorrência do comportamento sexualmente agressivo (Hall \& Barongan, 1997). Por sua vez, a teoria da aprendizagem social entende a violação primariamente como uma função das influências culturais que fomentam papéis de género restritivos, onde a sexualidade coerciva é legitimada. Isto é, os indivíduos assimilam motivos, atitudes e outras racionalizações que alimentam o comportamento sexualmente coercivo. A violência sexual é, assim, encarada como um comportamento aprendido, proveniente das interacções ambientais e rotinas sociais. Neste sentido, alguns autores têm explorado a relação entre masculinidade, atitudes sexuais e experiências precoces de exposição à violência. De facto, vários autores têm afirmado que a associação entre o género masculino e a agressão sexual é particularmente forte em sujeitos provenientes de ambientes familiares violentos, nos quais observaram violência entre os progenitores (e.g., Forbes \& Adams-Curtis, 2001) e/ou foram vítimas de abuso sexual (e.g., Gidycz et al., 1993). De forma menos directa, outros investigadores (e.g., Blumenthall, Neemann \& Murphy, 1998; Mohr, 1999, cit. Hunter, Figueredo, Malamuth \& Becker, 2004) sugerem que a exposição dos jovens à violência doméstica poderá ser um factor potenciador de vários problemas de comportamento, entre os quais a agressão sexual. A severidade destes problemas parece estar relacionada com a extensão da exposição à violência, bem como com o suporte parental recebido (Grych, Jouriles, Swank, McDonald \& Norwood, 2000, cit. ibidem). Também no estudo de Forbes e Adams-Curtis (2001), foi possível perceber a existência de uma importante relação entre violência na família de origem e a experiência de agressão e coerção sexual, quer para o sexo masculino, quer para o sexo feminino.

Contudo, um estudo muito recente (Loh \& Gidycz, 2006), baseado em relatos retrospectivos e prospectivos, questiona a relação entre estas duas variáveis. Ainda que mediante uma análise retrospectiva os autores tenham confirmado a relação entre vitimização sexual na infância e perpetração de comportamento sexual coercivo na idade adulta, os dados prospectivos não corroboram estes resultados. Desta forma, os autores consideram que esta relação poderá ser mediada por outros factores (e.g., estratégias de resolução dos conflitos familiares, estratégias de resolução de conflitos no namoro) e menos linear do que o inicialmente pensado. Destaque-se que, neste estudo, em apenas 5\% dos casos com vitimação sexual na infância se registou uma história de agressão sexual na idade adulta. 
De modo análogo, apesar da relevância teórica e empírica que tem vindo a ser atribuída às variáveis atitudinais, alguns estudos (e.g., Forbes \& Adams-Curtis, 2001; Vogel \& Himelein, 1995) não encontraram correlações significativas entre os mitos associados à violação e a agressão sexual, fazendo supor que a relação entre estas variáveis poderá não ser tão robusta como o assumido. A título meramente exemplificativo, refira-se que, no estudo de Vogel e Himelein (1995) atrás citado, das quatro variáveis atitudinais consideradas - representação conflitual da sexualidade, conservadorismo sexual, legitimação da violência interpessoal e legitimação de mitos sexuais -, apenas as três primeiras revelam impacto, e mesmo esse reduzido, na explicação da violação no contexto de namoro.

Para além das questões da socialização, os traços constitucionais e de personalidade dos agressores têm sido igualmente conceptualizados como potenciais preditores da vitimação sexual (Marx et al., 1996). Assim, Maletzky (2000, cit. Carr \& Van Deusen, 2004) considera que os factores genéticos poderão desempenhar um papel importante na predisposição para a violência sexual, considerando que a transmissão intergeracional de determinados traços de personalidade leva certos homens a reagir agressiva e impulsivamente. Mais especificamente, numa revisão recente sobre o tema (cf. Hunter et al., 2004), a hostilidade masculina surge como preditor significativo da vitimação sexual. Os homens que atingem níveis elevados de hostilidade tendem a adoptar determinados estereótipos e mitos sexuais e, deste modo, legitimam mais facilmente a violência interpessoal. É certo, contudo, que nem todos os estudos sobre esta hipótese corroboram tal interpretação (Hunter et al., 2004).

O desconhecimento do carácter ilícito dos comportamentos abusivos, por parte de muitos adolescentes perpetradores, emerge igualmente como preditor da agressão sexual. A este nível, sabe-se que, à medida que o conhecimento legal acerca da violência sexual aumenta, diminui a probabilidade de ofensa sexual (Maxwell et al., 2003).

Finalmente, de entre os vários factores situacionais associados pela literatura à violência sexual, o uso de álcool e/ou drogas constituem os preditores usualmente mais citados. Pese embora o facto de os diferentes estudos variarem na explicação da acção destas variáveis enquanto agentes desencadeadores da agressão sexual, todos eles enfatizam a sua potencialidade na predição deste tipo de abuso (Abbey, McAuslan, Zawacki, Clinton \& Buck, 2001; Gross et al., 2006; Himelein, 1995; Norris et al., 1996; Vogel \& Himelein, 1995). A investigação desenvolvida neste domínio comprova que cerca de metade dos casos de agressões sexuais estão associados ao consumo de álcool do ofensor, da vítima ou de ambos (e.g., 
Abbey et al., 1996; Green \& Navarro, 1997; Muhehlenhard \& Linton, 1987).

Adicionalmente, tem sido documentado que o consumo de álcool está associado a agressões sexuais mais severas e a uma maior probabilidade de estas agressões culminarem em violações (e.g., Abbey, Clinton-Sherrod, McAuslan, Zawacki, Clinton \& Buck, 2003; Abbey et al., 1996). Os estudos com base em relatos retrospectivos masculinos (Ullman, Karabatsos \& Koss, 1999a) e femininos (Ullman et al., 1999b) comprovam que o uso de álcool desempenha um papel directo e indirecto nos resultados da agressão sexual. Assim, no estudo com a amostra de homens, verificou-se que o consumo de álcool pela vítima antes do incidente abusivo exerce directa e indirectamente um contributo na severidade da agressão sexual, enquanto que o uso masculino de álcool apenas contribui para as consequências da agressão (Ullman et al., 1999a). No estudo com a amostra de mulheres, o consumo de álcool pela vítima e ofensor, previamente à experiência abusiva, contribui directa e indirectamente para a severidade da vitimação sexual, enquanto que o comportamento alcoólico feminino também contribui, ainda que em menor escala, para as consequências da agressão sexual. No entanto, o consumo de álcool pela vítima e ofensor, antes do episódio abusivo, diferem na predição dos resultados da vitimação: o uso de álcool pelo ofensor surge associado a mais agressividade e a uma vitimização sexual mais severa; por sua vez, os ofensores tornam-se menos agressivos perante vítimas que consumiram álcool, dado que, nestes casos, a força não é necessária para a concretização da ofensa sexual. Além disso, as vítimas nestas circunstâncias tendem a autoculpabilizar-se pelo sucedido, havendo uma menor responsabilização do agressor (Ullman et al., 1999a).

Ainda na explicação desta relação, outros autores (e.g. Breitenbecher, 2000; Norris et al., 1996) consideram que o consumo de álcool poderá diminuir a capacidade de detecção de risco por parte das vítimas e, deste modo, impedi-las de manifestar resistência à ofensa sexual. Por sua vez, o álcool poderá constituir tanto um precipitante como um inibidor do comportamento sexualmente agressivo do homem (e.g. Berkowitz, 1992, cit. Carr \& Van Deusen, 2004), o que tem vindo a contribuir para que a relação entre álcool e agressão sexual seja entendida de forma multifacetada.

A par destes factores situacionais, outros têm sido elencados na literatura como possuindo um contributo importante na predição da agressão sexual. Especificamente, o conhecimento que o ofensor possui da vítima, o isolamento do contexto, a existência anterior de actividade sexual consentida e a distorção masculina do grau de interesse feminino no acto sexual tendem a favorecer a ocorrência do assalto sexual (Abbey et al., 2001). 


\section{Impacto nas vítimas}

A par dos estudos sobre a prevalência e etiologia da agressão sexual, outros, ainda que em menor número, têm surgido no sentido de clarificar o impacto deste tipo de vitimização. Ainda que seja difícil estimar a longo prazo as consequências desta experiência, a literatura (e.g., Jackson \& Davis, 2000; Jezl, Molidor \& Wrigth, 1996; Koss, Dinero, Siebel \& Cox, 1988; Shapiro \& Schwarz, 1997) enumera um conjunto de sintomas que, frequentemente, são exteriorizados pelas vítimas, nomeadamente, humilhação, ansiedade, depressão, problemas de abuso de substâncias, perda de auto-estima, isolamento social, raiva, medo de contrair doenças sexualmente transmissíveis, vergonha e disfunções sexuais, entre outros. A ideação suicida parece constituir uma consequência igualmente presente em casos de vitimação sexual. Uma revisão da literatura levada a cabo por Ullman (2004), na tentativa de analisar uma eventual relação entre vitimização sexual feminina e comportamentos suicidas, ilustra que a grande maioria das tentativas de suicídio feminino $(70 \%)$ são atribuídas a desordens psiquiátricas, $12 \%$ à violação sexual e $7 \%$ à molestação. A autora apurou que a vitimização sexual feminina parece estar relacionada com tentativas de suicídio, através de uma grande variedade de processos, incluindo sequelas psiquiátricas, tais como a depressão e a PTSD, surgindo ainda frequentemente associada a comportamentos de risco (e.g., associação com pares delinquentes e/ou abuso de substâncias). Também Ackard e Neumark-Sztainer (2002) apuraram que 50\% dos sujeitos (masculinos e femininos) com experiências de violência no namoro e violação evidenciavam mais tentativas de suicídio do que os participantes femininos (12,5\%) e masculinos $(5,4 \%)$ que nunca passaram por este tipo de experiências. Adicionalmente, os autores concluíram que a violência no namoro e a violação também surgem associadas às desordens alimentares, à baixa auto-estima e a algum comprometimento ao nível do bem-estar emocional.

Outras evidências empíricas (e.g., Rothbaum, Foa, Riggs, Murdock \& Walsh, 1992, cit. Sochting et al., 2004) sustentam que cerca de $90 \%$ das vítimas adultas tendem a evidenciar sintomatologia congruente com a PTSD na semana seguinte à ocorrência do abuso e $47 \%$ continuam a exibir a mesma sintomatologia decorridos 9 meses da experiência abusiva. Outros (e.g. Kilpatrick et al., 1987, cit. ibidem) asseveram mesmo que os sintomas poderão persistir um ano após a ofensa sexual, tendo isto sido comprovado em cerca de $15 \%$ dos casos de vitimação.

Uma lacuna central no conhecimento acerca do impacto da vitimação sexual situa-se ao nível das autopercepções sexuais femininas. Um estudo particularmente interessado em compreender a relação entre estas duas variáveis comprova que a experiência de algum tipo de abuso, incluindo 
coerção sexual, durante o namoro, poderá suscitar na mulher autopercepções sexuais negativas que, por sua vez, surgem frequentemente associadas a uma baixa da auto-estima e sintomas depressivos (Offman \& Matheson, 2004). Também Shapiro e Schwarz (1997) sugerem que, a par da ansiedade, depressão e constrangimento psicológico, as vítimas de violação no namoro tendem a manifestar sintomas sexuais relacionados com o trauma (e.g., comportamento sexual disfuncional e outras preocupações sexuais, experiências dissociativas, memórias intrusivas, evitamento) e baixa auto-estima sexual em determinadas áreas, como sejam o julgamento moral (a avaliação que faz da moralidade do seu comportamento sexual e sentimentos), a adaptação (a avaliação da compatibilidade da sua sexualidade com outros objectivos da sua vida) e o controlo sexual (dos sentimentos, comportamentos e relações).

Salienta-se ainda, que, de acordo com alguns autores (e.g., Jackson \& Davis, 2000), o impacto da experiência de violência sexual poderá sentir-se também em outras pessoas para além da vítima, designadamente familiares e outros significativos, podendo estes experimentar sentimentos de raiva, culpa, sensação de perda e défices ao nível do ajustamento sexual. Estas reacções frequentemente interferem com o suporte prestado às vítimas primárias, impedindo-as de receber dos seus companheiros e familiares o apoio de que precisariam para uma boa recuperação.

\section{A cultura de prevenção da violência sexual: objectivos, alvos, conteúdos e eficácia}

Os indicadores de prevalência e impacto da vitimação sexual perpetrada nas relações amorosas atestam a imperativa necessidade de se proceder a uma intervenção precoce sobre este problema. As primeiras referências a tais programas de prevenção da violência nas relações amorosas surgem na década de 90 e, desde então, tem-se assistido a uma proliferação do investimento científico nesta área. Genericamente, estes programas visam consciencializar a população juvenil da gravidade e impacto da violência e promover comportamentos nãoviolentos nas relações íntimas, procurando diminuir a probabilidade de os jovens se tornarem, futuramente, ofensores ou vítimas (Suderman, Jaffe \& Hastings, 1995).

Mais especificamente, os programas de prevenção da violência sexual, geralmente conduzidos nas universidades e em outros contextos considerados de risco, como as escolas secundárias, integram como objectivos primordiais a modificação de crenças e atitudes sexuais que tendem a potenciar a probabilidade de os homens perpetrarem violência sexual e a restringir a capacidade de autodefesa das mulheres (Söchting et al., 2004). 
Efectivamente, de entre os vários conteúdos temáticos presentes nos diferentes programas de prevenção da violência sexual, as atitudes e crenças associadas à agressão sexual são aqueles que assumem um maior destaque. Exemplificativo disto mesmo é o facto de um conjunto de programas se centrar exclusivamente na alteração de atitudes legitimadoras da violação ou apenas avaliarem a sua eficácia pela modificação do grau de adesão a mitos referentes à violação (e.g., Lonsway \& Fitzgerald, 1994).

Do ponto de vista das estratégias, a grande maioria dos programas de prevenção da violência sexual na intimidade envolve uma grande diversidade, tais como o recurso a vídeos educacionais e discussões de grupo (Hanson \& Gidycz, 1993), a produções teatrais (Jaffe, Sudermann, Reitzel \& Killip, 1992, cit. Kuffel \& Katz, 2002), a prestação de informação sobre os direitos nas relações amorosas ou a educação para a mudança de crenças normativas em torno da violência e estereótipos de género. Tipicamente, procura-se também promover competências de resolução de conflitos (Avery-Leaf et al., 1997), divulgar os recursos de apoio às vítimas existentes na comunidade, incrementar a intolerância ao fenómeno e fomentar comportamentos apropriados de prevenção ou de resposta à agressão sexual (Weisz \& Black, 2001).

Em termos de alvos, os programas de prevenção nesta área podem dirigir-se apenas a participantes de sexo feminino, procurando clarificar a natureza da agressão sexual e os direitos das vítimas, ou apenas a participantes de sexo masculino, partindo do pressuposto de que os homens que sustentam menos mitos sexuais apresentam menos probabilidade de ser sexualmente agressivos (Schwartz \& DeKeseredy, 1997). Adicionalmente, embora com menor frequência, estes programas podem envolver audiências de ambos os géneros, tipicamente integrando informação em torno dos mitos sobre a violação e estereótipos sobre os papéis sexuais, a visualização de vídeos envolvendo cenários sexuais e dinamizando grupos de discussão sobre estas temáticas (Parrot \& Bechhofer, 1991, cit. Yeater \& O'Donohue, 1999). De referir que a literatura nesta área sustenta claramente a preferência por intervenções homogéneas (isto é, envolvendo um único sexo), já que sendo os homens geralmente os agentes abusivos e as mulheres as vítimas, as questões a analisar, bem como as técnicas a utilizar, deverão diferir em função do género (Lonsway, 1996). Tem sido defendido, deste modo, que os programas que integram só grupos de homens ou só grupos de mulheres revelam resultados mais promissores que os programas que envolvem audiências mistas.

De um modo geral, os programas de prevenção da violência sexual têm seguido duas direcções centrais. A primeira, que podemos designar como uma abordagem didáctica centrada na modificação de atitudes, engloba programas tipicamente em formato de workshop educacional com 
duração reduzida (1-2 horas), apoiando-se na asserção de que uma diminuição das atitudes que tendem a legitimar a violência sexual poderá implicar uma diminuição da incidência deste tipo de abuso. Este tipo de programas pode aglomerar várias componentes, especificamente, informação acerca da prevalência da violência sexual, desmistificação de mitos associados à problemática, discussão de papéis de género estereotipados e ainda sugestões práticas acerca dos comportamentos protectores a adoptar nas relações íntimas. Outros (cf. Breitenbecher, 2000) incluem também a identificação do risco de violência nas relações amorosas e a indução de empatia para com as vítimas de violação. Nesta linha inserimos alguns programas estritamente dirigidos ao sexo masculino, com especial ênfase na socialização dos papéis sexuais, e que pretendem, entre outros objectivos, ajudar os participantes a perceber e empatizar com as opiniões e decisões dos outros, facilitar a identificação das forças culturais que podem contribuir para a legitimação da agressão sexual e ainda ajudá-los a discriminar sexo consensual de sexo coercivo (Davis, 2000).

Paralelamente, a literatura descreve a emergência de programas, essencialmente direccionados para o sexo feminino, com o intuito de promover estratégias de autodefesa e treino de assertividade (Brecklin \& Ullman, 2005) e confrontação com uma potencial agressão (Söchting et al., 2004). Pese embora a escassez de estudos que comprovem a eficácia deste tipo de programas, os que existem têm mostrado que a implementação deste tipo de estratégias constitui uma das formas mais bem sucedidas de resistência à agressão sexual (Brecklin \& Ulman, 2005). Alguma polémica existe, contudo, sobre as formas de resistência específicas que devem ser treinadas nestes programas. Efectivamente, podemos distinguir duas abordagens de confronto, uma mais verbal e outra mais física. A primeira tem recebido maior consenso, nomeadamente a partir de autores (e.g., Muehenhard \& Linton, 1987) que sustentam que a violência sexual é, frequentemente, consequência de uma comunicação pobre e de percepções e expectativas desajustadas quanto ao comportamento do parceiro. Evocam, deste modo, a necessidade de treinar uma comunicação sexual assertiva, no sentido de promover uma adequada manifestação das expectativas sexuais sem ferir susceptibilidades no parceiro amoroso, fortalecendo a comunicação e a intimidade.

Por sua vez, outros autores enfatizam a utilidade de uma abordagem preventiva que aumente a capacidade de resistência física das mulheres em caso de ataque, sendo cada vez maior a oferta de cursos de autodefesa exclusivamente para o público feminino. Esta abordagem é, contudo, mais polémica, nomeadamente no seio do próprio movimento feminista. Analisando esta questão, Burton (1998) considera que o discurso feminista, com a preocupação de contestar a ideia de que as mulheres que não lutam con- 
sentiram na violação, tem alimentado a imagem da mulher paralisada pelo medo e incapaz de resistir. Embora se compreenda que a validação da reacção de medo e imobilidade seja necessária, até pela frequência com que de facto ocorre, também é um facto que este tipo de discurso ignora que as mulheres são capazes de lutar fisicamente, não o fazendo porque são treinadas ao longo da sua socialização para não o fazer e porque são levadas a acreditar que tal resultará em maior dano. Burton (1998) contesta precisamente esta ideia, citando vários estudos que evidenciam que o uso da resistência física, conjuntamente com outras formas de resposta (gritar, fugir), diminui a probabilidade de a violação se consumar. Esta não é, contudo, uma posição consensual e outros autores consideram que o envolvimento das mulheres neste tipo de programas as poderá tornar mais vulneráveis à agressão física concomitante à vitimação sexual, bem como à acusação de não terem interrompido o acto sexual, quando o poderiam ter feito.

Mais recentemente, outras propostas preventivas têm emergido (cf. McMahon, 2000), nomeadamente a ideia de que os esforços preventivos nesta área se devem apoiar, em primeiro lugar, no modelo de saúde públi$\mathrm{ca}^{8}$. Esta abordagem apresenta quatro fases distintas e sequenciais. Numa primeira fase, intitulada de averiguação da saúde pública, pretende-se recolher, analisar e interpretar os dados de saúde, seguindo-se a identificação dos factores de risco para a vitimização e perpetração. Posteriormente e ambicionando o conhecimento da eficácia das acções preventivas, é promovida a realização de estudos acerca do impacto da implementação dos programas e, por último, decorre a disseminação da informação acerca do que deles resulta, podendo isto ser feito de diferentes formas (e.g., jornais, internet).

Efectivamente, muito embora se tenha assistido nos últimos anos a uma propagação de programas de prevenção da violência sexual nas relações amorosas, o mesmo não tem sucedido no que concerne a avaliações que ilustrem a eficácia e/ou impacto dos mesmos. Primariamente centrados nas mudanças das atitudes sobre a violência sexual, os programas de prevenção raramente compreendem a avaliação da sua efectiva eficácia na diminuição da prevalência do fenómeno (e.g., Lonsway \& Fitzgerald, 1995; Lonsway, 1996). Assim, a grande maioria das avaliações do impacto deste

\footnotetext{
${ }^{8}$ Este modelo de saúde pública repousa em três tipos de prevenção: primária, secundária e terciária (Prothrow-Stith, Spivak \& Sege, 1997, cit. McMahon, 2000). A prevenção terciária compreende o trabalho com homens e mulheres que perpetraram repetidamente ofensas sexuais. A um nível secundário, a prevenção direcciona-se para indivíduos que tenham iniciado recentemente as ofensas sexuais, pretendendo-se a sua identificação precoce e subsequente tratamento. Por último, a prevenção primária visa, essencialmente, barrar o desenvolvimento do problema, ou seja, impedir que os indivíduos considerados em risco possam vir a perpetrar este tipo de vitimação sexual (cit. ibidem).
} 
tipo de programas assume como sinal de eficácia a mudança ao nível atitudinal, nomeadamente na legitimação de mitos sexuais, nas atitudes face à violação, na aceitação da violência interpessoal e nas atitudes face à mulher (Breitenbecher, 2000).

Estas avaliações tendem a apontar para resultados positivos deste tipo de acções preventivas na modificação das atitudes legitimadoras da agressão sexual (e.g., Breitenbecher, 2000; Kuffel \& Katz, 2002; Yeater and O' Donohue, 1999; Pinzone-Glover et al., 1998; Weisz \& Black, 2001), tal como é comprovado pela revisão exaustiva dos resultados dos programas conduzida por Lonsway (1996). Outros autores têm, contudo, encontrado resultados menos optimistas, sobretudo quando consideram também o impacto específico a nível comportamental, na redução efectiva das agressões sexuais (Breitenbecher \& Gidycz, 1998; Hanson \& Gidycz, 1993; Pinzone-Glover, Gidycz \& Jacobs, 1998).

Começamos por destacar Hanson e Gidycz (1993), os primeiros a conduzir um dos mais sofisticados estudos, em termos metodológicos, sobre a eficácia de um programa de prevenção conduzido com um grupo de 360 estudantes do sexo feminino. Pretendia-se a consciencialização sobre o fenómeno, a diminuição da aceitação de mitos sexuais, a sensibilização para o impacto que determinadas variáveis sociais têm sobre a vitimação sexual, a promoção de estratégias para lidar com situações de risco, a alteração de comportamentos de risco, a promoção de uma comunicação sexual clara e efectiva nas relações de namoro e a diminuição da incidência da violência sexual durante um período de 9 semanas. Este estudo demonstrou-se, sobretudo, eficaz no aumento dos conhecimentos sobre a problemática e verificou-se uma redução da incidência do problema entre mulheres sem história de agressão sexual. Não se encontraram, contudo, resultados positivos entre mulheres com história de vitimação sexual. Na sequência deste estudo, outros (e.g., Breteinbecher \& Gidycz, 1998; Breitenbecher \& Scarce, 1999) emergiram, indicando a falta de eficácia deste tipo de programas na diminuição da incidência da agressão sexual, em mulheres com ou sem história de vitimação sexual.

Este tipo de resultados mistos encontrados na avaliação da eficácia dos programas tem sido relacionado com a inconsistência e falta de uniformidade que alguns dizem existir nos próprios projectos preventivos. Efectivamente, os programas diferem em termos de conteúdos, no tipo de audiência que integram, no sexo dos administradores dos programas, no tempo que decorre entre a administração do programa e aplicação de medidas de avaliação do seu impacto (Pinzone-Glover et al., 1998). Por outro lado, alguns autores, mesmo que relativamente optimistas perante o impacto da prevenção na violência sexual (e.g., Breitenbecher, 2000), sugerem que determinadas variáveis, incluindo o género dos participantes e a sua história de vitimização sexual constituem importantes mediadores dos resultados deste tipo de esforços preventivos. 
Em suma, vários estudos comprovam a relevância dos programas até agora desenvolvidos na alteração atitudinal e modificação das crenças legitimadoras de violência. Como áreas mais polémicas, destaca-se a utilidade ou não de incluir nestes programas preventivos o treino de autodefesa para as mulheres, assim como o relativo desconhecimento que ainda existe sobre a eficácia dos programas implementados no plano comportamental.

\section{Limitações dos programas e propostas para futuras investigações}

Para além das áreas-problema que acabámos de assinalar, outras limitações têm sido apontadas aos programas de prevenção. Na verdade, se a participação nestes parece resultar em atitudes menos legitimadoras da agressão sexual, são ainda parcas as informações sobre os processos cognitivos, emocionais e comportamentais inerentes a estas mudanças (Breitenbecher, 2000). Outras limitações metodológicas e conceptuais (e.g., não integração de modelos teóricos no desenvolvimento dos programas, ausência de medidas psicométricas adequadas para determinar o impacto, recurso a amostras tendenciosas e outras questões de desejabilidade social) têm sido apontadas, o que dificulta ainda mais o alcance de resultados conclusivos quanto à sua eficácia (cf. Lonsway, 1996; Yeater \& O’Donohue, 1999).

Por outro lado, sabe-se que a grande maioria dos programas de prevenção tem sido dirigida ao sexo feminino, na tentativa de reduzir a sua vulnerabilidade. Todavia, os especialistas da área reconhecem que a redução do risco da vitimação sexual feminina não deverá ser o focus primário, atendendo a que são os homens os principais agentes da grande maioria da violência sexual (e.g., Rozee \& Koss, 2001, cit. Carr \& Van Deusen, 2004). Face a esta realidade social, torna-se imprescindível a realização de mais esforços preventivos direccionados para os potenciais perpetradores desta forma de violência. A este nível, investigações realizadas sobre a avaliação de programas de prevenção orientados para o sexo masculino, estabelecem que as mudanças constatadas são imediatas, ou seja, a curtoprazo, desconhecendo-se os seus efeitos a longoprazo, dado que raros são os estudos que realizam follow up. Deste modo, torna-se difícil estimar o tempo de duração destas mudanças e, consequentemente, o impacto efectivo destes esforços preventivos na redução da incidência das ofensas sexuais (Lonsway, 1996). Desta forma, o desenvolvimento de estudos prospectivos seria útil para um conhecimento mais profundo da manutenção no tempo das alterações atitudinais (Breitenbecher, 2000).

Torna-se ainda importante notar que, apesar de a grande maioria dos estudos sobre a prevalência da violência sexual nas relações amorosas apontar para uma superioridade da vitimação feminina, começa a tornar-se claro 
que os homens também podem ser vitimizados. Neste sentido, é fundamental a emergência de estudos que clarifiquem a prevalência real da perpetração feminina deste tipo de violência, as suas motivações e dinâmicas.

Ainda no que concerne às amostras estudadas, a elevada prevalência da violência sexual entre estudantes universitários torna fundamental que se desencadeiem mais variados esforços preventivos junto desta população. Contudo, há quem defenda que a violência sexual é um problema com início precoce, na adolescência, pelo que importa apostar também na prevenção junto dos grupos etários mais novos (Himelein, 1995; Humphrey \& White, 2000).

Importa ainda advertir que estes esforços preventivos devem envolver outras pessoas que não apenas os estudantes universitários. Como Power e colaboradores (2000, cit. Banyard et al., 2004) defendem, a mera existência de programas de prevenção da violência sexual não é, por si só, suficiente. Estas acções preventivas devem abarcar todos os elementos da comunidade estudantil, num trabalho que permita veicular uma mensagem de não tolerância face a este tipo de violência. Só assim será possível uma consolidação efectiva das aprendizagens resultantes deste tipo de acções.

Num plano mais macro, autores como Jewkes (2002) defendem que em determinados contextos, a violação é uma estratégia, culturalmente aprovada, de controlo e de disciplina da mulher. Sustentam, assim, a necessidade de promover o estatuto social da mulher e de alterar as normas que regem os papéis de género. Neste sentido, Feltey e colaboradores (1991) destacam a importância de introduzir nos programas de prevenção referências específicas às dinâmicas de socialização de género, às desigualdades estruturais entre homens e mulheres, assim como à relação entre sexo e violência.

Tal não significa, contudo, como vimos, que a diminuição das atitudes legitimadoras da violação sexual garanta a subsequente redução da incidência deste tipo de vitimação. Assim, outros consideram que, mais do que apostar na modificação das atitudes, os programas de prevenção deverão focar-se primeiramente na informação e competências de autoprotecção para as potenciais vítimas, capacitando as mulheres para a identificação de situações abusivas, consciencializando-as das consequências dos hábitos etílicos e treinando competências de comunicação, essencialmente a assertividade (Söchting et al., 2004). Especificamente no que concerne ao álcool, e atendendo a que o seu consumo (pela vítima e pelo ofensor) tem sido consensualmente associado a um maior risco de agressão sexual, alguns autores (e.g., Abbey et al., 2001) defendem a necessidade de os esforços preventivos contemplarem esta matéria, usualmente esquecida nestes programas.

Por último, e tendo em conta a realidade portuguesa, caracterizada pela escassez de estudos sobre a prevalência e incidência desta forma especifica de abuso nas relações íntimas, bem como pela parca cultura de pre- 
venção neste domínio, seria útil que entre nós se começasse a produzir um maior investimento científico nesta matéria, quer no plano da sua caracterização, quer da prevenção.

\section{Referências}

Abbey, A., Clinton-Sherrod, A. M., McAuslan, P., Zawacki, T., Clinton, A. M., \& Buck, P. O. (2003). The relationships between the quantity of alcohol consumed and the severity of sexual assaults committed by college men. Journal of Interpersonal Violence, 18, 813-833.

Abbey, A., McAuslan, P., Zawacki, T., Clinton, A. M., \& Buck, P. O. (2001). Attitudinal, experiential and situational predictors of sexual assault perpetration. Journal of Interpersonal Violence, 16, 784-807.

Abbey, A., Ross, L. T., McDuffie, D., \& McAuslan, P. (1996). Alcohol and dating risk factors for sexual assault among college women. Psychology of Women Quarterly, 20, 147-169.

Ackard, D. M. \& Neumark-Sztainer (2002). Date violence and date rape among adolescents: associations with disordered eating behaviors and psychological health. Child Abuse \& Neglect, 26, 455-473.

Associação Portuguesa de Apoio à Vítima (2004). Relatório de actividades do projecto IUNO. Lisboa: APAV.

Avery-Leaf, S., Cascardi, M. A., O’Leary, K. D., \& Cano, A. (1997). Efficacy of a dating violence prevention program on attitudes justifying aggression. Journal of Adolescent Health, 21, 11-17.

Banyard, V. L., Plante, E. G., \& Moynihon, M. M. (2004). Bystander education. Bringing a broader community perspective to sexual violence prevention. Journal of Community Psychology, 32, 61-79.

Becker, J. V., \& Keilly, D. W. (1999). Preventing sexual abuse and assault. Sexual Abuse: A Journal of Research and Treatment, 11, 267-278.

Bergman, L. (1992). Dating violence among high school students. Social Work, 37, 21-27.

Brecklin, L. R., \& Ullman, S. E. (2005). Self-defense or assertiveness training and women's responses to sexual attacks. Journal of Interpersonal Violence, 20, 738-762.

Breitenbecher, K. H. (2000). Sexual assault on college campuses: Is an ounce of prevention enough?. Applied \& Prevention Psychology, 9, 23-52.

Breitenbecher, K. H., \& Gidycz, C. A. (1998). An empirical evaluation of a program designed to reduce the risk of multiple sexual victimization. Journal of Interpersonal Violence, 13, 472-488.

Breiteinbecher, K. H., \& Scarce, M. (1999). A longitudinal evaluation of the effectiveness of a sexual assault education program. Journal of Interpersonal Violence, 14, 459-478. 
Burton, N. (1998). Resistance to prevention: Reconsidering feminist antiviolence rhetoric. In S. French, W. Teays \& L. Purdy (Eds.), Violence against women. Philosophical perspectives (pp. 182-200). Ithaca: Cornell University Press.

Carr, J. L., \& Van Deusen, K. M. (2004). Risk factors for male sexual aggression on college campuses. Journal of Family Violence, 19, 279-289.

Cook, S. L. (1995). Acceptance and expectation of sexual aggression in college students. Psychology of Women Quarterly, 19, 181-194.

Davis, T. (2000). Programming for men to reduce sexual violence. New Directions for Student Services, 90, 79-89.

Feltey, K. M., Ainslie, J. J., \& Geib, A. (1991). Sexual coercion attitudes among high school students. The influence of gender and rape education. Youth \& Society, 23, 229-250.

Foshee, V. A. (1996). Gender differences in adolescent dating abuse prevalence, types and injuries. Health Education Research, 11, 275-286.

Forbes, G. B., \& Adams-Curtis, L. E. (2001). Experiences with sexual coercion in college males and females. Role of family conflict, sexist attitudes, acceptance of rape myths, self-esteem, and the big-five personality factors. Journal of Interpersonal Violence, 16, 865-889.

Gabinete de Estudos e Planeamento do Ministério da Justiça (1997). Estatísticas criminais. Lisboa: GEPMJ.

Gabinete de Estudos e Planeamento do Ministério da Justiça (1998). Estatísticas da justiça. Lisboa: GEPMJ.

Geiger, B., Fischer, M., \& Eshet, Y. (2004). Date-rape-supporting and victim-blaming attitudes among high school students in a multiethnic society. Journal of Interpersonal Violence, 19, 406-426.

Gidycz, C. A., Coble, A. N., Latham, L., \& Layman, M. (1993). Sexual assault experience in adulthood and prior victimization experiences. A prospective analysis. Psychology of Women Quarterly, 17, 151-168.

Gidycz, C. A., Hanson, K., \& Layman, M. (1995). A prospective analysis of the relationships among sexual assault experiences. An extension of previous findings. Psychology of Women Quarterly, 19, 5-29.

Green, D. M., \& Navarro, R. L. (1998). Situation-specific assertiveness in the epidemiology of sexual victimization among university women. A prospective path analysis. Psychology of Women Quarterly, 22, 589-604.

Gross, A. M., Winslett, A., Roberts, M., \& Gohm, C. L. (2006). An examination of sexual violence against college women. Violence Against Women, 12, 288-300.

Hall, G. C. N., \& Barongam, C. (1997). Prevention of sexual aggression. Sociocultural risk and protective factors. American Psychologist, 52, 5-14.

Hanson, K. A., \& Gidycz, C. A. (1993). Evaluation of sexual assault prevention program. Journal of Consulting and Clinical Psychology, 61, 1046-1051.

Harned, M. S. (2002). A multivariate analysis of risk markers for dating violence victimization. Journal of Interpersonal Violence, 17, 1179-1197.

Hickman, L. J., Jaycox, L. H., \& Aronoff, J. (2004). Dating violence among adolescents: prevalence, gender distribution and prevention program effectiveness. Trauma, Violence \& Abuse, 5, 123-142. 
Himelein, M. J. (1995). Risk factors for sexual victmization in dating. A longitudinal study of college women. Psychology of Women Quarterly, 19, 31-48.

Humphrey, J. A., \& White, J. W. (2000). Women's vulnerability to sexual assault form adolescence to young adulthood. Journal of Adolescent Health, 27, 419-424 .

Hunter, J. A., Figueredo, A. J., Malamuth, N. M., \& Becker, J. (2004). Developmental pathways in youth sexual aggression and delinquency: Risk factors mediators. Journal of Family Violence, 19, 233-242.

Jackson, T. L., \& Davis, J. L. (2000). Prevention of sexual and physical assault toward women: A program for male athletes. Journal of Community Psychology, 28, 589-605.

Jackson. S. M., Cram, F., \& Seymour, F. W. (2000). Violence and sexual coercion in high school student's dating relationships. Journal of Family Violence, 15, 23-36.

Jezl, D., Molidor, C., \& Wright, T. (1996). Physical, sexual and psychological abuse in high school dating relationships outcomes: a comparison of the self-esteem. Child and Adolescent Social Work Journal, 13, 69-87.

Jewkes, R. (2002). Preventing sexual violence: A rights-based approach. The Lancet, 360, 1092-1093.

Koss, M. P., \& Cleveland, H. H. (1997). Stepping on toes: Social rotes of date rape lead to intractability and politicization. In Martin D. Schwartz (Ed.), Researching sexual violence against women: methodological and personal perspectives (4-22). Thousand Oaks: Sage Publications.

Koss, M. P., Dinero, T. E., Seibel, C. A, \& Cox, S. L. (1988). Stranger and acquaintance rape. Are there differences in the victim's experience? Psychology of Women Quarterly, 12, 1-24.

Kuffel, S. W., \& Katz, J. (2002). Preventing physical, psychological and sexual aggression in college dating relationships. The Journal of Primary Prevention, $22,361-374$.

Lanier, C. A. (2001). Rape-accepting attitudes: Percursors to or consequences of forced sex. Violence Against Women, 7, 876-885.

Loh, C., \& Gidycz, C. A. (2006). A prospective analysis of the relationships between childhood sexual victimization and perpetration of dating violence and sexual assault in adulthood. Journal of Interpersonal Violence, 21, 732-749.

Loh, C., Gidycz, C. A., Lobo, T. R., \& Luthra, R. (2005). A prospective analysis of sexual assault perpetration. Risk factors related to perpetrator characteristics. Journal of Interpersonal Violence, 20, 1325-1348.

Lonsway, K. A. (1996). Preventing acquaintance rape through education. What do we know? Psychology of Women Quarterly, 20, 229-265.

Lonsway, K. A., \& Fitzgerald, L. F. (1994). Rape myths: In review. Psychology of Women Quarterly, 18, 133-164.

Lonsway, K. A., \& Fitzgerald, L. F. (1995). Atitudinal antecedents of rape myth acceptance: A theoretical and empirical reexamination. Journal of Personality and Social Psychology, 68, 704-711. 
Machado, C., Matos, M., \& Moreira, A. I. (2003). Violência nas relações amorosas: Comportamentos e atitudes na população universitária. Psychologica, 33, 69$-83$.

Marx, B. P., Victoria, V. W., \& Gross, A. M. (1996). Date rape risk factors: A review and methodological critique of the literature. Aggression and Violent Behavior, 1, 27-45.

Maxwell, C. D., Robinson, A. L., \& Post, L. A. (2003). The nature and predictors of sexual victimization and offending among adolescents. Journal of Youth and Adolescence, 32, 465-477.

Mahoney, P., Williams, L. M., \& West, C. M. (2001). Violence against women by intimate relationships partners. In C. M. Renzetti, J. L. Edleson \& R. K. Bergen (Eds.), Sourcebook on violence against women (pp. 143-178). Thousand Oaks: Sage Publications.

McMahon, P. (2000). The public health approach to the prevention of sexual violence. Sexual Abuse: A Journal of Research and Treatment, 12, 27-26.

Muehlenhard, C. L., \& Linton, M. A. (1987). Date rape and sexual aggression in dating situations: incidence and risk factors. Journal of Counseling Psychology, 34, 186-196.

Muehlenhard, C. L., Friedman, D. E., \& Thomas, C. M. (1985). Is date justifiable? The effects of dating activity, who iniated, who paid, and men's attitudes toward women. Psychology Women Quarterly, 9, 297-310.

Muram, D., Hostetler, B. R., Jones, C. E., \& Speck, P. M. (1995). Adolescent victims of sexual assault. Journal of Adolescent Health, 17, 372-375.

Norris, J., Nurius, P. S., \& Dimeff, L. A. (1996). Through her eyes: factors affecting women's perception of and resistance to acquaintance sexual aggression threat. Psychology of Women Quarterly, 20, 123-145.

Paiva, C., \& Figueiredo, B. (2004). Abuso no relacionamento íntimo: Estudo de prevalência em jovens adultos portugueses. Psychologica, 36, 75-107.

Offman, A., \& Matheson, K. (2004). The sexual self-perceptions of young women experiencing abuse in dating relationships. Sex Roles, 51, 551-560.

Pinzone-Glover, H. A., Gidycz, C. A., \& Jacobs, C. D. (1998). An acquaintance rape prevention program. Effects on attitudes toward women, rape-related attitudes, and perceptions of rape scenarios. Psychology of Women Quarterly, 22, 605-621 .

Poitras, M., \& Lavoie, F. (1995). A study of the prevalence of sexual coercion in adolescent heterosexual dating relationships in a Quebec Sample. Violence and Victims, 10, 299-313.

Russell, B. L., \& Oswald, D. L. (2001). Strategies and dispositional correlates of sexual coercion perpetrated by women: An exploratory investigation. Sex Roles, 45, 103-115.

Rozee, P. D. (1993). Forbidden or forgiven? Rape in cross-cultural perspective. Psychology of Women Quarterly, 17, 499-514.

Sanders, B., \& Moore, D. (1999). Childhood maltreatment and date rape. Journal of Interpersonal Violence, 14, 115-124. 
Schwartz, M. D., \& DeKeseredy, W. S. (1997). Sexual assault on the college campus. The role of male peer support. Thousand Oaks: Sage Pulications.

Schubot, D. B. (2001). Date rape prevalence among female high school students in a rural Midwestern state during 1993, 1995, and 1997. Journal of Interpersonal Violence, 16, 291-196.

Serquino-Ramiro, L. (2005). Physical intimacy and sexual coercion among adolescent intimate partners in the Philippines. Journal of Adolescent Research, 20, 476-496.

Shapiro, B. L., \& Schwarz, J. C. (1997). Date rape. Its relationship to trauma symptoms and sexual self-esteem. Journal of Interpersonal Violence, 12, 407-419.

Söchting, I., Fairbrother, N., \& Koch, W. J. (2004). Sexual assault of women: Prevention efforts and risk factors. Violence Against Women, 10, 73-93.

Straus, M. A. (2004). Prevalence of violence against dating partners by males and female university students worldwide. Violence against women, 10, 790-811.

Suderman, M, Jaffe, P. G., \& Hastings, E. (1995). Violence prevention programs in secondary (high) schools. In E. Peled, P. G. Jaffe \& J. L. Edleson (Eds.), Ending the cicle of violence. Community responses to children of battered women (pp. 232-274). Thousand Oaks: Sage Publications.

Truman, D. M., Tokar, D. M., \& Fisher, A. R. (1996). Dimensions of masculinity: Relations to date rape supportive attitudes and sexual aggression in dating situations. Journal of Counselling and Development, 74, 555-562.

Ullman, S. E. (2004). Sexual assault victimization and suicidal behaviour in women: A review of the literature. Aggression and Violent Behavior, 9, 331-351.

Ullman, S. E., Karabatsos, G., \& Koss, M. (1999a). Alcohol and sexual aggression in a national sample of college men. Psychology Women Quarterly, 23, 673 -689 .

Ullman, S. E., Karabatsos, G., \& Koss, M. (1999b). Alcohol and sexual assault in a national sample of college women. Journal of Interpersonal Violence, 14, 603-625 .

Vicary, J. R., Klingaman, L. R., \& Harkness, W. L. (1995). Risk factors associated with date rape and sexual assault of adolescent girls. Journal of Adolescence, 18, 289-306.

Vogel, R. E., \& Himelein, M. J. (1995). Dating and sexual victimization: an analysis of risk factors among precollege women. Journal of Criminal Justice, 23, 153 -162 .

Yeater, E. A., \& O’ Donohue, W. (1999). Sexual assault prevention programs: Current issues, future directions and the potential efficacy of interventions with women. Clinical Psychology Review, 19, 739-771.

Weisz, A. N., \& Black, B. M. (2001). Evaluating a sexual assault and dating violence prevention program for urban youths. Social Work Research, 25, 89-100 .

Wilson, A. E., Calhoun, K. S., \& Bernat, J. A. (1999). Risk recognition and trauma-related symptoms among sexually revictimized women. Journal of Consulting and Clinical Psychology, 67, 705-710. 